\title{
A Conserved Linearization Approach for Solving Nonlinear Oscillation Problems
}

\author{
Sıla Ö. Korkut ${ }^{1, *}$, Nurcan Gücüyenen Kaymak ${ }^{2}$ and Gamze Tanoğlu ${ }^{2}$ \\ ${ }^{1}$ Department of Engineering Science, Izmir Katip Çelebi University, Izmir 35620, Turkey \\ ${ }^{2}$ Department of Mathematics, Izmir Institute of Technology, Izmir 35430, Turkey
}

Received: 19 Feb. 2018, Revised: 2 Apr. 2018, Accepted: 11 Apr. 2018

Published online: 1 May 2018

\begin{abstract}
Nonlinear oscillation problems are extensively used in engineering and applied sciences. Due to non-availability of the analytic solutions, numerical approaches have been used for these equations. In this study, a numerical method which is based on Newton-Raphson linearization and Fréchet derivative is suggested. The convergence analysis is also studied locally. The present method is tested on three examples: damped oscillator, Van-der Pol equation and Schrödinger equation. It is shown that the obtained solutions via the present method are more accurate than those of the well-known second order Runge-Kutta method. When examining the present method, preservation of characteristic properties of these equations is also considered. The obtained results show that the present method is applicable with respect to the efficiency and the physical compatibility.
\end{abstract}

Keywords: Fréchet derivative, Newton-Raphson method, Conservative scheme, Nonlinear oscillations, Linearization technique.

\section{Introduction}

Solving nonlinear oscillation problems has a key role in applied sciences and engineering because many engineering problems arise from vibrating systems which can be modeled by oscillation systems, see $[15,6,4]$. It is difficult to describe the exact solution for the nonlinear problems. Therefore, researchers have developed several methods to find approximate solutions of such equations. For example, homotopy analysis methods [10], harmonic balance method [14], homotopy perturbation method [8] etc.

Moreover, a great deal of effort has been spent for developing the numerical solutions of nonlinear oscillation problems. Differential transform method is used in [16], Taylor matrix method has been improved for solving the Duffing equation in [2]. Operator splitting methods are used in [13] and [17]. The iterative splitting method is used to solve nonlinear oscillation problems in [9]. A compact finite difference scheme has been applied to nonlinear Schrödinger equation in [3]. Another technique which is based on finding periodic solutions has been proposed for second and fourth order Duffing equation in [7].

Numerical methods produce approximated solutions. However, it is expected from the numerical schemes to satisfy some qualitative properties of the exact solution. This paper concerns with a linearization method for solving nonlinear oscillation problems which conserves the qualitative properties over a long time. We linearize the nonlinear equations by using Newton-Raphson process and Fréchet derivative approximation. This technique is originated in the study of Liu \& Wu, [11], for solving ordinary differential equations(ODEs) of Duffing-type nonlinearity. It can also be seen in [11,12,5]. In Ref. [5], which is our main reference, the authors applied the technique to the systems of differential equations. The Fréchet derivative is used to convert a nonlinear differential equation into a linear one iteratively. In the mentioned studies, the authors combine Fréchet derivative with the generalized differential quadrature rule(GDQR). In this work, Fréchet derivative is combined with the central difference approximation. Additionally, the applicability of the method on the partial differential equation is also controlled.

The paper is organized as follows: Section 2 provides the introduction to proposed method for the systems of differential equations. In addition, the convergence issue of the method is discussed, locally. In Section 3, we consider three main oscillation systems which are known in engineering and applied sciences. In order to check the accuracy of the present method a damped oscillator is first studied numerically. For this purpose, second order Runge-Kutta method (RK2) which is a well-known method among engineers and scientists is used for comparing the results. As our second example, Van-der Pol equation is considered to see the effects of the present method on the ordinary differential equation. Finally, as a partial

\footnotetext{
*Corresponding author e-mail: silaovgu.korkut@ikc.edu.tr
} 
differential equation, Schrödinger equation is studied to check the validity of the present method. Section 4 is dedicated to our brief conclusion.

\section{Local convergence analysis of the method}

This section is associated with both the analysis and the application of the method. After employing the convergence analysis locally, we will give a brief introduction for the application of the method to the nonlinear differential equation. A general nonlinear equation is given as follows:

$N(U)=0$

where $N$ includes differential operators. By the Newton-Raphson process, the solution of (1) is

$U^{n+1}=U^{n}+\theta^{n}$.

Here $n$ corresponds to the iteration number and $\theta^{n}$ corresponds to the refinement variable for correcting function $U^{n}$. Primarily, for solving refinement variable, we deal with the following differential equation

$\theta^{n} N^{\prime}\left(U^{n}\right)+N\left(U^{n}\right)=0$.

By definition of Fréchet derivative the term $\theta^{n} N^{\prime}\left(U^{n}\right)$ is defined as follows:

$\theta^{n} N^{\prime}\left(U^{n}\right)=\left.\frac{\partial}{\partial \varepsilon} N\left(U^{n}+\varepsilon \theta^{n}\right)\right|_{\varepsilon=0}$.

\subsection{Convergence analysis}

Let $X$ and $Y$ be Banach spaces and $L: \Omega \subseteq X \rightarrow Y$ be nonlinear operator given in Equation (1) in an open domain $\Omega_{0} \subseteq \Omega$. We concentrate on the following method:

$\theta^{n+1}=\theta^{n}-N^{\prime}\left(\theta^{n}\right)^{-1} N(U)$

Assumption 2.1.1 Let $N: \Omega_{0} \subseteq X \rightarrow Y$ be a Fréchet differentiable operator given in Equation (1). Assume that $\alpha>0, \beta>0$ and $\gamma>0$ are real numbers satisfying that there exists a fixed point $x^{*} \in \Omega_{0}$ for every $x, y \in \Omega_{0}$ yields:

$$
N\left(x^{*}\right)=0, \quad N^{\prime}\left(x^{*}\right)^{-1} \in N(X, Y),
$$

$\left\|N^{\prime}\left(x^{*}\right)^{-1}\left(N^{\prime}(x)-N^{\prime}\left(x^{*}\right)\right)\right\| \leq \alpha\left\|x-x^{*}\right\|$,

$\left\|N^{\prime}\left(x^{*}\right)^{-1}\left(N^{\prime}(x)-N^{\prime}(y)\right)\right\| \leq \beta\|x-y\|$.

We also introduce a helpful function as follows:

$h(s)=\frac{\beta s}{2} \frac{1}{1-\alpha s}$.

Let us choose $0 \leq s<\frac{1}{\alpha+\beta / 2}$, then

$0 \leq h(s)<1$.

Under this assumption, the following theorem describes the local convergence analysis of the method.

Theorem 2.1.2 Let $N: \Omega_{0} \subseteq X \rightarrow Y$ be a Fréchet differentiable operator given in Equation (1). Assume that $\alpha>0, \beta>0$ and $\gamma>0$ are real numbers satisfying that there exists a fixed point $x^{*} \in \Omega_{0}$. Then the method defined by (5) is well defined for every $\theta^{n} \in B\left(\theta^{*}, \frac{1}{\alpha+\beta / 2}\right)$ where $n=0,1,2, \ldots$ and converges to $\theta^{*}$.

Proof 2.1.2 We start with assumption given in (6) i.e. $\left\|N^{\prime}\left(\theta^{*}\right)^{-1}\left(N^{\prime}\left(\theta^{n}\right)-N^{\prime}\left(\theta^{*}\right)\right)\right\| \leq \alpha\left\|\theta^{n}-\theta^{*}\right\| \leq 1$

where $\theta^{n} \in \Omega_{0}$. Remember the following remark:
Remark.Let $B$ be a Banach algebra. If $\|x\| \leq 1$ then $1-x$ is invertible with

$\left\|(1-x)^{-1}\right\| \leq \frac{1}{1-\|x\|}$.

After substituting $N^{\prime}\left(\theta^{n}\right)^{-1} N^{\prime}\left(\theta^{*}\right)-1$ in place of $x$, then $N^{\prime}\left(\theta^{n}\right)^{-1}$ exists and we obtain:

$\left\|N^{\prime}\left(\theta^{n}\right)^{-1} N^{\prime}\left(\theta^{*}\right)\right\| \leq \frac{1}{1-\alpha\left\|\theta^{n}-\theta^{*}\right\|}$.

Consider the method such as

$\theta^{n+1}=\theta^{n}-N^{\prime}\left(\theta^{n}\right)^{-1} N(U)$.

Subtracting $\theta^{*}$ from Equation (13) and then using Lagrange identity, we obtain:

$$
\begin{aligned}
\theta^{n+1}-\theta^{*} & =\theta^{n}-\theta^{*}-N^{\prime}\left(\theta^{n}\right)^{-1} N(U) \\
& =-N^{\prime}\left(\theta^{n}\right)^{-1}\left(N(U)-N^{\prime}\left(\theta^{n}\right)\left(\theta^{n}-\theta^{*}\right)\right) \\
& =-L^{\prime}\left(\theta^{n}\right)^{-1} L^{\prime}\left(\theta^{*}\right) \int_{0}^{1} L^{\prime}\left(\theta^{*}\right)^{-1} \chi\left(\theta^{*}, \theta\right)\left(\theta^{n}-\theta^{*}\right) d t
\end{aligned}
$$

where $\chi\left(\theta^{*}, \theta\right)=\left(N^{\prime}\left(\theta^{*}+t\left(\theta^{n}-\theta^{*}\right)\right)-N^{\prime}\left(\theta^{n}\right)\right)$ and $N(U)=$ $N\left(\theta^{n}\right)=0$. After taking norms and using (6) we obtain:

$$
\begin{aligned}
\left\|\theta^{n+1}-\theta^{*}\right\| & \leq\left\|N^{\prime}\left(\theta^{n}\right)^{-1} N^{\prime}\left(\theta^{*}\right)\right\| \cdot \\
& \left\|\int_{0}^{1} N^{\prime}\left(\theta^{*}\right)^{-1} \chi\left(\theta^{*}, \theta\right)\left(\theta^{n}-\theta^{*}\right) d t\right\| \\
& \leq \frac{1}{1-\alpha\left\|\theta^{n}-\theta^{*}\right\|} \cdot \frac{\gamma}{2}\left\|\theta^{n}-\theta^{*}\right\|^{2} \\
& \leq h\left(\left\|\theta^{n}-\theta^{*}\right\|\right)\left\|\theta^{n}-\theta^{*}\right\|<\left\|\theta^{n}-\theta^{*}\right\| .
\end{aligned}
$$

To express $\theta^{n}$ in terms of $\theta^{0}$, we substitute $n=0,1, \ldots n$ and get the following

$\left\|\theta^{n+1}-\theta^{*}\right\| \leq h\left(\left\|\theta^{n}-\theta^{*}\right\|\right)^{n+1}\left\|\theta^{0}-\theta^{*}\right\|$,

where $\left\|\theta^{n}-\theta^{*}\right\| \in B\left(\theta^{*}, \frac{1}{\alpha+\beta / 2}\right)$.

Then by taking the limit such that $\lim _{n \rightarrow \infty} h\left(\left\|\theta^{n}-\theta^{*}\right\|\right)^{n+1}=0$ we have $\lim _{n \rightarrow \infty} \theta^{n+1}=\theta^{*}$ which means that recommended method converges to the solution.

\subsection{Application of the method}

Since the convergence of the method is guaranteed, it can be applied to any nonlinear differential equations. In this study we mainly focus on the systems of nonlinear oscillatory systems. Thus, as for the systems of differential equations, the Equation (1) turns to

$N_{1}(U, V)=0$,

$N_{2}(U, V)=0$.

The Newton-Raphson method is applied for both variables given in Equation (17). It yields

$U^{n+1}=U^{n}+\theta_{1}^{n}$,

$V^{n+1}=V^{n}+\theta_{2}{ }^{n}$,

with the iteration number $n$. Here, $\theta_{1}{ }^{n}$ and $\theta_{2}{ }^{n}$ are the refinements. When $\theta_{1}{ }^{n}$ and $\theta_{2}{ }^{n}$ are approaching to zero, $U_{1}$ and $U_{2}$ are approximating to their fixed points. Thus, finding the 
values of $\theta_{1}{ }^{n}$ and $\theta_{2}{ }^{n}$ have essential role in determining the solution. To get these values the following equation will be solved in each iteration.

$\theta_{1}{ }^{n} N_{1}{ }^{\prime}\left(U^{n}, V^{n}\right)+\theta_{2}{ }^{n} N_{1}{ }^{\prime}\left(U^{n}, V^{n}\right)+N_{1}\left(U^{n}, V^{n}\right)=0$,

$\theta_{1}{ }^{n} N_{2}{ }^{\prime}\left(U^{n}, V^{n}\right)+\theta_{2}{ }^{n} N_{2}{ }^{\prime}\left(U^{n}, V^{n}\right)+N_{2}\left(U^{n}, V^{n}\right)=0$

where $\theta_{i}{ }^{n} N_{j}{ }^{\prime}\left(U^{n}, V^{n}\right), i, j=1,2$ is obtained by the help of Fréchet derivatives. Then,

$\theta_{1}{ }^{n} N_{i}{ }^{\prime}\left(U^{n}, V^{n}\right)=\left.\frac{\partial}{\partial \varepsilon} N_{i}\left(U^{n}+\varepsilon \theta_{1}{ }^{n}, V^{n}\right)\right|_{\varepsilon=0}$
$\theta_{2}{ }^{n} N_{i}{ }^{\prime}\left(U^{n}, V^{n}\right)=\left.\frac{\partial}{\partial \varepsilon} N_{i}\left(U^{n}, V^{n}+\varepsilon \theta_{2}{ }^{n}\right)\right|_{\varepsilon=0}$.

Crank-Nicolson scheme is implemented to the Equation (20) to describe the solutions. To be more understandable of the expression, the application of the present method to the nonlinear oscillation problems will be shown in the next section.

\section{Numerical tests and simulations}

The main purpose of this section is to test the efficiency of the proposed method for some numerical examples and to check whether the method preserves the characteristic properties of the equations.

Example 3.1. To see the performance of the method, the damped oscillator is considered as a test problem. However, before giving the results, we shall show how the method is applied to any systems of differential equations.

$q^{\prime \prime}+f\left(q^{\prime}\right)+q=0$,

where $f\left(q^{\prime}\right)=\alpha\left(q^{\prime}\right)^{3}$ and $\alpha$ denotes the damping constant. By redefining the variables, the second order equation is turned to the first order system of differential equation

$$
\begin{aligned}
q_{1}{ }^{\prime}-q_{2} & =0, \\
q_{2}{ }^{\prime}+q_{1}+\alpha q_{2}{ }^{3} & =0
\end{aligned}
$$

where $q(t)=q_{1}(t)$ and $q^{\prime}(t)=q_{2}(t)$. Thus, two systems of equations are obtained as follows:

$N_{1}\left(q_{1}, q_{2}\right)=q_{1}{ }^{\prime}-q_{2}$

$N_{2}\left(q_{1}, q_{2}\right)=q_{2}^{\prime}+q_{1}+\alpha q_{2}{ }^{3}$.

For sake of clarity, we only deal with the time interval $[0, h]$ in introducing the algorithm. By using the algorithms given in (19) and (20), we obtain

$$
\begin{gathered}
N_{1}\left(q_{1}, q_{2}\right)=\left.\frac{\partial}{\partial \varepsilon}\left[q_{1}^{\prime}+\varepsilon \theta_{1}^{\prime}-q_{2}\right]\right|_{\varepsilon=0}+\left.\frac{\partial}{\partial \varepsilon}\left[q_{1}^{\prime}-q_{2}-\varepsilon \theta_{2}\right]\right|_{\varepsilon=0} \\
N_{2}\left(q_{1}, q_{2}\right)=\left.\frac{\partial}{\partial \varepsilon}\left[q_{2}^{\prime}+\left(q_{1}+\varepsilon \theta_{1}\right)+\alpha\left(q_{2}\right)^{3}\right]\right|_{\varepsilon=0} \\
+\left.\frac{\partial}{\partial \varepsilon}\left[q_{2}^{\prime}+\varepsilon \theta_{2}{ }^{\prime}+q_{1}+\alpha\left(q_{2}+\varepsilon \theta_{2}\right)^{3}\right]\right|_{\varepsilon=0} .
\end{gathered}
$$

As a result,

$$
\begin{aligned}
\theta_{1}{ }^{\prime}-\theta_{2}+q_{1}{ }^{\prime}-q_{2} & =0 \\
\theta_{2}{ }^{\prime}+\theta_{1}+3 \alpha q_{2}{ }^{2} \theta_{2}+q_{2}{ }^{\prime}+q_{1}+\alpha q_{2}{ }^{3} & =0 .
\end{aligned}
$$

\begin{tabular}{|c|c|c|c|c|c|}
\hline \multirow[b]{2}{*}{$\alpha$} & \multirow[b]{2}{*}{ Method } & \multirow[b]{2}{*}{$h$} & \multicolumn{3}{|c|}{ Norms } \\
\hline & & & $L_{\infty}$ & $L_{2}$ & $L_{1}$ \\
\hline 0.0001 & $\begin{array}{l}\text { The present method } \\
\text { RK2 }\end{array}$ & $\begin{array}{l}2^{-3} \\
2^{-3} \\
\end{array}$ & $\begin{array}{l}0.0011 \\
0.0020 \\
\end{array}$ & $\begin{array}{l}0.0017 \\
0.0030 \\
\end{array}$ & $\begin{array}{l}0.0038 \\
0.0065 \\
\end{array}$ \\
\hline 0.0001 & $\begin{array}{l}\text { The present method } \\
\text { RK2 }\end{array}$ & $\begin{array}{l}2^{-4}-4 \\
2^{-4}\end{array}$ & $\begin{array}{l}2.9783 \mathrm{e}-004 \\
5.1439 \mathrm{e}-004 \\
\end{array}$ & $\begin{array}{c}\begin{array}{c}6.1720 \mathrm{e}-004 \\
0.0010\end{array} \\
\end{array}$ & $\begin{array}{l}0.0020 \\
0.0030\end{array}$ \\
\hline 0.0001 & $\begin{array}{l}\text { The present method } \\
\text { RK2 }\end{array}$ & $2^{2-5}$ & $\begin{array}{l}9.2573 \mathrm{e}-005 \\
1.1844 \mathrm{e}-004\end{array}$ & $\begin{array}{l}2.8095 \mathrm{e}-004 \\
2.9516 \mathrm{e}-004\end{array}$ & $\begin{array}{l}0.0013 \\
0.0012\end{array}$ \\
\hline
\end{tabular}

Table 1: Numerical errors for different $\Delta t$. Estimated errors using $L_{\infty}, L_{2}$ and $L_{1}$ norms are obtained comparing with the analytic solution.

After rearranging the equations, it corresponds to

$$
I_{2} \Theta_{t}+A \Theta+B(\Theta, \Lambda)=-I_{2} \Lambda_{t}-A \Lambda-C(\Lambda),
$$

where $\Theta=\left(\theta_{1}, \theta_{2}\right)^{T}, \Lambda=\left(q_{1}, q_{2}\right)^{T}$,

$$
A=\left[\begin{array}{cc}
0 & -1 \\
1 & 0
\end{array}\right], B(\Theta, \Lambda)=\left[\begin{array}{c}
0 \\
3 \alpha q_{2}{ }^{2} \theta_{2}
\end{array}\right] \text { and } C(\Lambda)=\left[\begin{array}{c}
0 \\
\alpha q_{2}{ }^{3}
\end{array}\right] \text {. }
$$

Moreover, $I_{2}$ is a $2 \times 2$ identity matrix. The system given in Equation (27) is linear with respect to $\Theta$. Thus, this system is solved for $\Theta$ by using Crank-Nicolson scheme. We have

$$
\begin{gathered}
I_{2} \frac{\Theta^{n+1}-\Theta_{n}}{\Delta t}+A \frac{\Theta^{n+1}+\Theta^{n}}{2}+D\left(\Theta_{n}^{n+1}, \Lambda_{n}^{n+1}\right)=-I_{2} \frac{\Lambda^{n+1}-\Lambda_{n}}{\Delta t} \\
-B \frac{\Lambda^{n+1}+\Lambda^{n}}{2}-C\left(\Lambda_{n}^{n+1}\right) .
\end{gathered}
$$

The problem is solved when the initial position is 1 and the initial velocity is zero. Here, the analytic solution of one-dimensional damped oscillator is

$q(t) \approx \frac{\cos t}{\sqrt{1+\frac{3 \alpha t}{4}}}$, where $\alpha \rightarrow 0^{+}$.

For details, it is referred to [1]

In Table 1, the errors are presented in different discrete norms, namely $L_{1}, L_{2}$ and $L_{\infty}$. Here, $L_{1}, L_{2}$ and $L_{\infty}$ norms are defined by

$$
\begin{aligned}
\|\cdot\|_{L_{1}} & =\sum_{n=0}^{n=N}|u(n h)-U(n h)|, \\
\|\cdot\|_{L_{2}} & =\left(\sum_{n=0}^{n=N}|u(n h)-U(n h)|^{2}\right)^{1 / 2} \\
\|\cdot\|_{L_{\infty}} & =\max _{0 \leq n \leq N}|u(n h)-U(n h)| .
\end{aligned}
$$

The numerical solution obtained by the present method is compared with the analytic one on the interval $[0,1]$ for various values of $\alpha$. Table 1 reveals that the proposed linearized method(PLM) is more efficient than the well-known second order Runge-Kutta method(RK2).

Figs 1 and 2 illustrate that there is no difference between the numerical solutions and the analytic solutions for $\alpha=0.1$ and $\alpha=0.001$, respectively, on $[0,30]$. It can also be seen from the figures that the amplitude of the Equation (21) is damping as $\alpha$ getting bigger. 


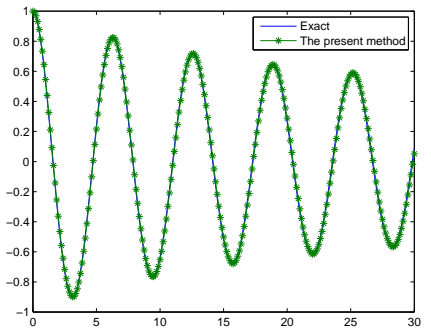

Fig. 1: Analytic solution vs. Numerical solution for $\alpha=0.1$

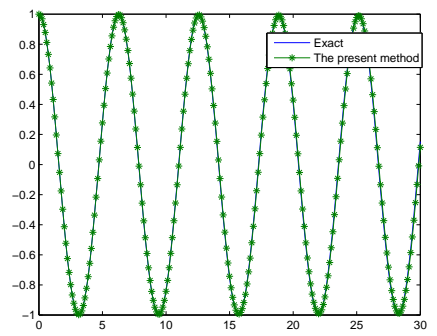

Fig. 2: Analytic solution vs. Numerical solution for $\alpha=0.001$

In Figs 3 and 4, $\alpha$ is taken as 1 in order to see the effects of nonlinearity on the present method. However, to the best our knowledge, there is no exact solution for the Equation (21) for the choice of $\alpha$. For this reason, the results obtained from the present method are compared with a known software in MATLAB, ODE45 which is known as an explicit solver. Figs 3 and 4 compare the solutions obtained by the present method with ODE45 and RK2. It is worth to say that the RK2 has the stability problem over a long time. We studied with $\Delta t=1 / 8$ on $[0,30]$. In Figure 3, it is obvious that the present method is in a perfect agreement with the ODE45 in MATLAB.

Example 3.2. As our second example we consider Van-der Pol equation which has a great deal of importance for engineers, physicists as well as mathematicians.

$\ddot{x}+\mu\left(x^{2}-1\right) \dot{x}+x=0$,

where $\mu$ denotes the constant of nonlinearity. Rearranging Equation (30) and employing the present method given in Section 2, we have

$$
I_{2} \Theta_{t}+A \Theta+D(X) \Theta=-I_{2} X_{t}-A X-C(X),
$$

where $\Theta=\left(\theta_{1}, \theta_{2}\right)^{T}, X=\left(x_{1}, x_{2}\right)^{T}$ and $I_{2}$ is the identity matrix. Here,

$$
\begin{array}{cc}
A=\left[\begin{array}{cc}
0 & -1 \\
1 & 0
\end{array}\right], & D(X)=\left[\begin{array}{cc}
0 & 0 \\
2 \mu x_{1} x_{2} & \mu\left(x_{1}^{2}-1\right)
\end{array}\right], \\
C(X) & =\left[\begin{array}{c}
0 \\
\mu\left(x_{1}^{2}-1\right) x_{2}
\end{array}\right] .
\end{array}
$$

To obtain Figs 5 and $6, \Delta t$ is taken as 0.01 over the interval $[0,60]$ where $\mu=5$ and $\mu=10$, respectively. The solutions of the present method are compared with the ODE23s code in

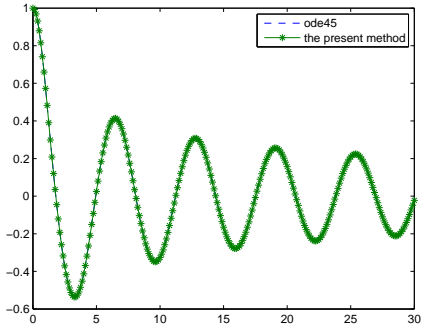

Fig. 3: Solutions of ODE45 and the present method

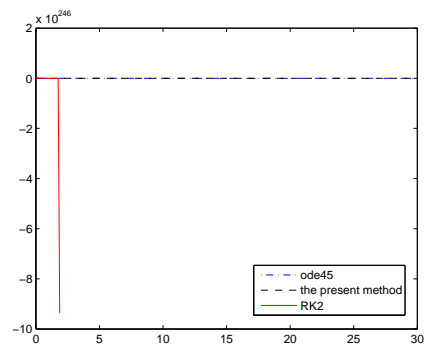

Fig. 4: Solutions of ODE45, RK2 and the present method
MATLAB since there is no exact solution for Equation (30). We note that the ODE23s is valid for the stiff and nonlinear problems. which means that ODE23s is an implicit solver in MATLAB. Although the present method is explicit, Figs 5 and 6 show that the present method is in a good agreement with ODE23s for different values of $\mu$. It is known that many explicit methods do not preserve some qualitative properties. Even though the present method is explicit, it preserves the limit cycle of Van-der Pol equation which is the most known qualitative property of the equation. Figure 7 emphasizes that the present method preserves the limit cycle of the equation in the case of $\mu=10$ where $t \in[0,60]$. For the chosen parameters, it is observed that the elapsed time is 0.2031 for the present method whereas the elapsed time is 1.4063 for the software of MATLAB, ODE23s, with the same number of steps on time. Although the difference is a small number, it will be important whenever the system is huge or multi-dimensional. Thus, we shall emphasize that the present method is faster than ODE23s in CPU runtime.

Example 3.3. One-dimensional cubic nonlinear Schrödinger equation(CNSE) which arises in the quantum physics and applied sciences is considered. The CNSE is defined as follows:

$\psi_{t}=\left(-\frac{1}{2} \frac{\partial^{2}}{\partial x^{2}}+\left(\frac{1}{1+\sin ^{2} x}+30 *|\psi|^{2}\right)\right) \psi$

subject to the Dirichlet boundary condition

$$
\psi\left(x_{L}, t\right)=\psi\left(x_{R}, t\right)=0,
$$

and the initial condition is taken

$$
\psi_{0}(x)=\gamma \exp (\sin 2 x),
$$




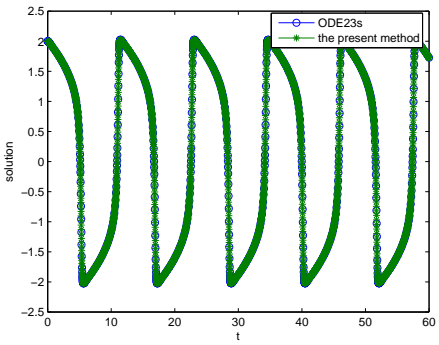

Fig. 5: Comparison of the solutions of the present method and ODE23s in the case of $\mu=5$.

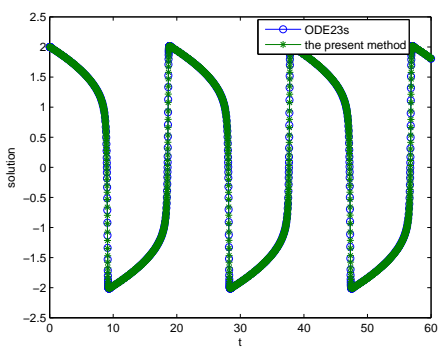

Fig. 6: Comparison of the solutions of the present method and ODE23s in the case of $\mu=10$.

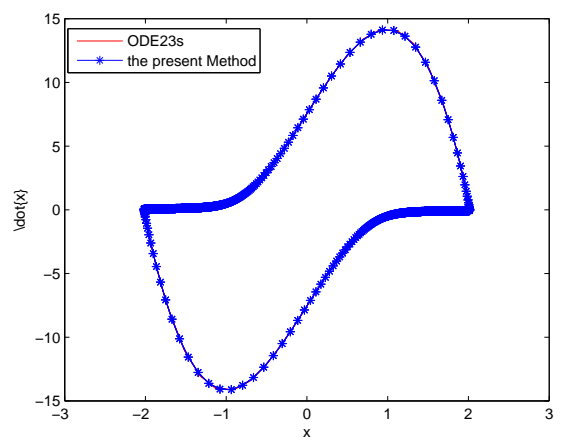

Fig. 7: Comparison of the obtained solutions by the present method and ODE23s to see the conservation of the limit cycle of the Equation (30). Here, $\mu=10$ and $t \in[0,60]$ with $\Delta t=0.01$.

where $\gamma$ represents the normalization constant.

Applying the present method and doing tedious calculations the system to be computed has arisen. The main objective of this example is to check the validity of the present method on the partial differential equations. For that purpose, the main focus is the conservation of the qualitative properties such as probability density of the particle and the mass conservation of the particle.

To compare the present method with the second order Runge-Kutta(RK2) method, we also study for $x \in[-20,20]$ where $N_{x}=N_{t}=100$. The system is integrated to the final time $t_{\text {end }}=4$. Figs 8 and 9 illustrate the numerical solutions of the present method and the RK2, respectively. It is obvious that the

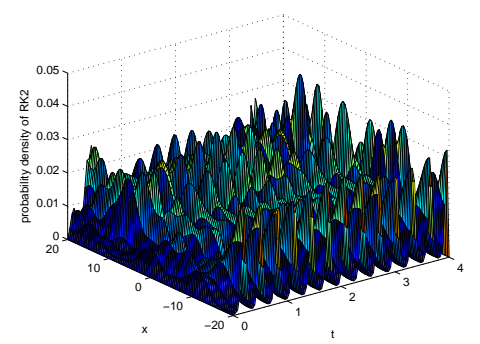

Fig. 8: Numerical Solution of Equation (32) obtained by RK2

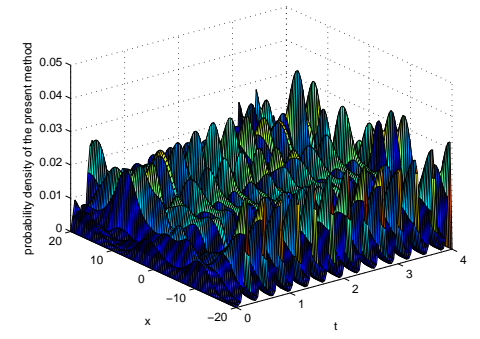

Fig. 9: Numerical solution of Equation (32) obtained by the present method

numerical solutions are so similar to each other, see Figs 8 and 9. However, when the final time is taken as $t_{\text {end }}=5$, it is observed that the RK2 does not preserve the probability density of the particle in Equation (32) for a long time.

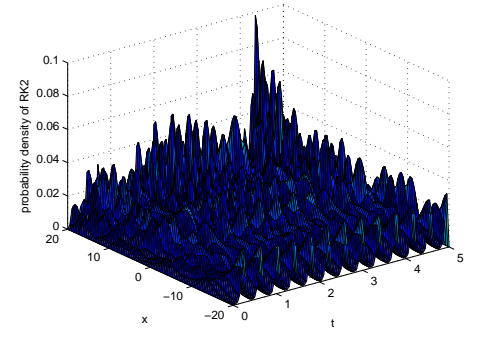

Fig. 10: The numerical solutions obtained from the RK2 method over $t \in[0,5]$.

The probability density of the particle should be conserved with respect to the conservation law. Figure 10 indicates that the RK2 does not confirm the law whereas in Figs 11 and 12 the 
present method preserves the probability density of the particle in Equation (32) even when the final time $t_{\text {end }}=8$.

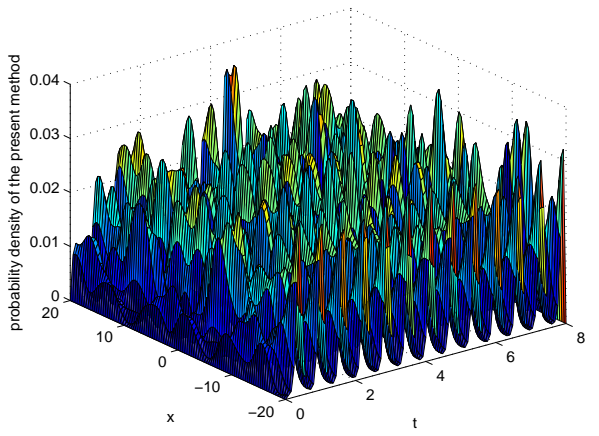

Fig. 11: Numerical Solution of Equation (32) obtained by the present method

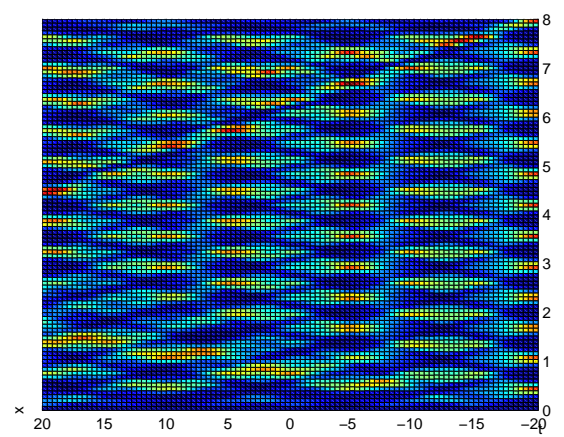

Fig. 12: Contour Plot of the numerical solution of Equation (32) obtained by the present method

Due to the aim of this study, to ensure the validity of the present method, checking conservation of the mass is necessary. To this end, the initial mass is computed as

$$
\int_{-20}^{20}|\psi|^{2} d x=0.4000
$$

while the numerical solutions obtained by the present method and RK2, respectively, at $t_{\text {end }}=4$

$$
\int_{-20}^{20}|\psi|^{2} d x=0.3968, \text { and } \int_{-20}^{20}|\psi|^{2} d x=0.4038
$$

At $t_{\text {end }}=7$ the observed results are

$$
\int_{-20}^{20}|\psi|^{2} d x=0.3913, \text { and } \int_{-20}^{20}|\psi|^{2} d x=N a N .
$$

These results guarantee that the present method has the long-time behavior for the conservation of the characteristic properties.

\section{Conclusions and discussions}

This numerical study introduces a linearization method whose iterative process is based on the Newton-Raphson process and Fréchet derivative. After ensuring that the method is convergent, the performance of the present method is tested on three main nonlinear oscillation problems. To check it, analytic solution and some numerical software in MATLAB are used as well as the second order Runge-Kutta method (RK2). It is observed that the present method conserves the qualitative properties of the equations. Moreover, it is more efficient than the compared methods. Even though the present method is explicit, it achieves a good agreement with an implicit solver, ODE23s, and the analytic solution. The RK2, which is a well-known method among the engineers, has some problems on the conservation of the qualitative problems and on the long-time behavior. As a result, the present method is applicable for nonlinear oscillation problems in applied sciences and engineering.

\section{Acknowledgement}

The authors thank the anonymous referee for a careful checking of the details and for helpful comments that improved this paper.

\section{References}

[1] C.M. Bender and S. A. Orszag, Advanced Mathematical Methods for Scientists and Engineers I, Springer Science \& Business Media, New York, (1999)

[2] B. Bülbül and M. Sezer, Numerical Solution of Duffing Equation by using an Improved Taylor Matrix Method, Journal of Applied Mathematics, Vol. 2013, 6 pages, doi: 10.1155/2013/691614, (2013).

[3] M. Dehghan and A. Taleei, A compact split-step finite difference method for solving the nonlinear Schrödinger equations with constant and variable coefficients, Computer Physics Communications, Vol. 181, No.1, pp. 43-51 (2010).

[4] A. D. Dimarogonas, Vibration for Engineers, Prentice Hall, New Jersey, (1996).

[5] M.R. Fazel, M.M. Moghaddam and J. Poshtan, Application of GDQ method in nonlinear manipulator undergoing large deformation, Proceedings of the Institution of Mechanical Engineers, Part C: Journal of Mechanical Engineering Science, Vol. 227, No. 12, pp. 2671-2685 (2013).

[6] A. Fidlin, Nonlinear Oscillations in Mechanical Engineering, Nonlinear Oscillations in Mechanical Engineering, Springer, Berlin, (2010).

[7] F. R. Groves JR., Numerical solution of nonlinear differential equation using computer algebra, International Journal of Computer Mathematics, Vol. 13, No. 3-4, pp. 301-309 (1983).

[8] J. H. He, Homotopy perturbation technique, Computer Methods in Applied Mechanics and Engineering, Vol. 178, No. (3-4), pp. 257-262 (1999).

[9] S. Ö. Korkut Uysal and G. Tanoğlu, An Efficient Iterative Algorithm for Solving Non-Linear Oscillation Problems, FILOMAT, Vol. 31, No. 9, pp. 2713-2726 (2017).

[10] S. J. Liao and A. T. Cheung, Application of homotopy analysis method in nonlinear oscillations, Journal of Applied Mechanics, Vol. 65, No. 4, pp. 914-922 (1998). 
[11] G. R. Liu and T. Y. Wu, Numerical solution for Differential Equations of Duffing-Type non-linearity using the Generalized differential Quadrature Rule, Journal of Sound and Vibration, Vol. 237, No. 5, pp. 805-817 (2000).

[12] G. R. Liu and T. Y. Wu, An application of the generalized differential quadrature rule in Blasius and Onsager equations, International Journal for Numerical Methods in Engineering, Vol. 52, No. 9, pp. 1013-1027 (2001).

[13] C. Lubich, On splitting Methods for Schrodinger-Poisson and cubic nonlinear Schrodinger Equations, Mathematics of computation, Vol. 77, No. 264, pp. 2141-2153 (2008).

[14] R. E. Mickens, Mathematical and numerical study of the Duffing-harmonic oscillators, Journal of Sound and Vibration, Vol. 244, No. 3, pp. 563-567 (2001).

[15] A. H. Nayfeh and D. T. Mook, Nonlinear Oscillations, WILEY-VCH Verlag GmbH \& Co. KGaA, (2004)

[16] K. Tabatabaei and E. Günerhan, Numerical Solution of Duffing Equation by the Differential Transform Method, Appl. Math. Inf. Sci. Lett., Vol. 2, No. 1, pp. 1-6 (2014).

[17] M. Thalhammer, High-Order Exponential Operator Splitting Methods for Time-Dependent Schrödinger Equations, SIAM J. Numer. Anal., Vol. 46, No. 4, pp. 2022-2038 (2008).

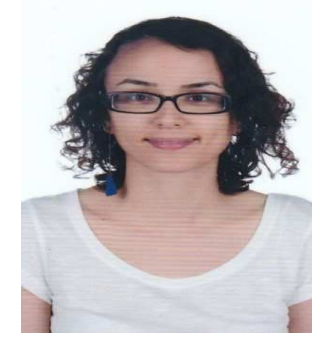

Sıla Ö. Korkut received the $\mathrm{PhD}$ degree in Mathematics at Izmir Institute of Technology. Her research interests are in the areas of applied mathematics and numerical methods for differential equations including construction of a method and analysis.

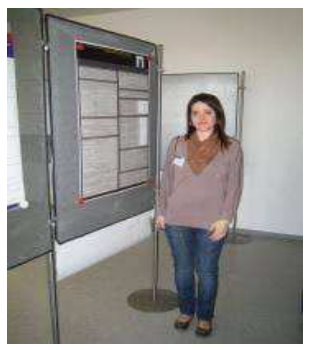

Nurcan Gücüyenen Kaymak got her $\mathrm{PhD}$ degree in Mathematics at Izmir Institute of technology. Her research interests are: applied mathematics and numerical methods for differential equations including construction of a method and analysis.

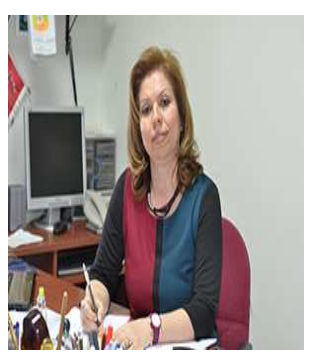

Gamze Tanoğlu is Proffesor in Mathematics at Izmir Institute of Technology. She received the PhD degree in "Applied Mathematics" at University of Delaware (USA) in 2000. She has published research articles in reputed international journals of mathematical and engineering sciences. Her research interests are in the areas of dynamical systems, applied mathematics and numerical methods for differential equations including construction of a method and analysis. 